\title{
Präparation und Charakterisierung von biologisch aktiven Magnetit-Protein-Nanopartikeln
}

\author{
Hans-Dieter Hunger, Katerina Vaskova
}

\section{Einleitung}

Magnetische Nanopartikel besitzen Anwendungspotenzial in vielen biologischen und medizinischen Anwendungsgebieten. Superparamagnetische Magnetit-Nanopartikel werden klinisch verwendet als Kontrastmittel im Magnet-Resonanz-Imaging (MRI)und intensiv untersucht für Anwendungen wie Drug-Delivery-Systeme, Zell-Bewegungs- und Trenn-Systeme und Hyperthermie (1-5). Dabei sind Eisenoxid (Magnetit)-Nanopartikel besonders interessant, da sie größenabhängigen Superparamagnetismus besitzen, nicht toxisch sind und im Organismus metabolisiert werden (5, 6). Deshalb werden unterdessen Magnetit-Nanopartikel in vielen biomedizinischen und diagnostischen Anwendungen eingesetzt (7-9). Die Stabilität und Biokompatibilität der Magnetit-Nanopartikel sind von der Größe der Partikel und von der Oberflächenbeschichtung der Partikel in der Lösung abhängig (10). Die häufigste Methode zur Herstellung von superparamagnetischen Magnetit-Nanopartikeln ist die Kopräzipitation von Eisensalzen in der Gegenwart einer Base, meist Ammoniumhydroxid (11). Für biomedizinische Anwendungen sollte die Oberfläche der Magnetit-Partikel komplett mit einer Polymerschicht überzogen sein, um den Eisenoxidkern gegen die Blutproteine und Phagocytose-Rezeptoren abzuschirmen (12). Die üblichsten Beschichtungsmoleküle für biokompatible, auf Magnetit basierende Kolloide sind Derivate von Dextran und Polyethylenglycol $(13,15)$.

In dieser Arbeit beschreiben wir eine neue Präparationsmethode von Magnetit-Protein-Partikeln durch direkte Kopräzipitation von Eisensalzen und Proteinen. Als Modellprotein wird Humanserumalbumin (HSA) verwendet, das als Hauptprotein des humanen Blutserums an der Oberfläche von Partikeln zur Verwendung in medizinischen Diagnostik- und Therapieverfahren sehr gute Voraussetzungen für die Biokompatibilität der MagnetitPartikel besitzt. Dabei wurde gefunden, dass die meisten der primären Präzipitationsprodukte zunächst biologisch inaktiv (z. B. nicht von HSA-spezifischen Antikörpern erkennbar) sind und erst durch weitere chemische Aktivierungsschritte biologisch aktiviert werden können. Das Verfahren der chemische Aktivierung und dessen Optimierung wird beschrieben und eine Modellvorstellung für diesen Aktivierungsschritt präsentiert.

Magnetit-Nanopartikel-Kopplungsprodukte mit unterschiedlichen Biomolekülen (z. B. Antikörpern, Protein A) gehören zu den für eine Verwendung in humanmedizinischen Strategien favorisierten Transportvehikeln für bioaktive Moleküle. Deshalb wurden die von uns erhaltenen Magnetit-HSA-Komplexe in einem zweistufigen Glutaraldehyd Verfahren chemisch aktiviert und kovalent mit Protein A und Antikörpern (IgG) gekoppelt.
Für die Bewertung der biologischen Aktivität der MagnetitProtein-Partikel wurde ein Festphasenassay unter Verwendung von Nitrocellulosemembranen als Trägermaterial und fluoreszenzmarkierten (FITC) immunologischen Nachweisreagenzien entwickelt und eingesetzt. Der Festphasenassay wurde mittels Photo-Imaging bzw. Laserscanner-ImagingTechnik ausgewertet und dokumentiert.

\section{Methoden und Materialien}

\subsection{Herstellung der Magnetit-Protein-Partikel}

Die Präparation der Magnetit-HSA-Partikel erfolgte durch Kopräzipitation von Eisensalzen mit HSA. Dabei wurden 1,3 ml einer Eisenstammlösung (Fe(II)-Chlorid, Fe(III)Chlorid, äquimolar) und 2mg HSA (in $1 \mathrm{ml}$ destilliertem Wasser) vermischt und mittels $0,5 \mathrm{ml}$ Ammoniak präzipitiert. Nach sofortiger Zugabe von ca. $40 \mathrm{ml}$ destilliertem Wasser und guter Vermischung (Vortex) wurden die magnetischen Komplexe mittel eines Magneten an der Röhrchenwand konzentriert und der Überstand dekantiert. Die Waschung wurde noch dreimal wiederholt und die Partikelpäparation in 4 Aliquots $(1 \mathrm{ml})$ geteilt. Präzipitierte Magnetit-Partikel ohne HSA im Fällungsansatz wurden als Kontrolle parallel hergestellt.

\subsection{Chemische Aktivierung der Magnetit- Protein-Partikel}

Zur chemischen Aktivierung wurden Aliquots der Magnetit-HSA-Partikel mit unterschiedlichen organischen Säuren (z. B. Zitronensäure, Weinsäure und Oxalsäure) bei $20^{\circ} \mathrm{C}$ ca. 30 Sekunden inkubiert, danach viermal mit destilliertem Wasser gewaschen und in $1 \mathrm{ml}$ Wasser resuspendiert. Die Inkubation erfolgte mittels unterschiedlicher Konzentrationen und Volumina der Säuren. Das Gesamtvolumen der magnetisch abgeschiedenen Magnetit-Protein-Partikel wurde vor und nach dem Säurebehandlungsschritt bestimmt.

\subsection{Festphasenassay zur Bestimmung der biologischen Aktivität}

$2 \mu \mathrm{l}$ der aliquotierten Magnetit-HSA-Partikel-Präparationen, einschließlich Verdünnungen, wurden auf Nitrocellulosestreifen aufgetüpfelt und angetrocknet. Auf den gleichen Streifen wurden Negativkontrollen (z. B. Magnetit-Partikel) und Positivkontrollen (z. B. HSA-Verdünnungen und fluoreszenzmarkierte Antikörper) getüpfelt. Nach einem Blockierungsschritt der Oberfläche des Trägermaterials mittels $0,1 \%(\mathrm{w} / \mathrm{v})$ Gelantine in PBS wurden die Streifen mit einem FITC markierten anti-HSA-Antikörper 3 Stunden inkubiert, mit PBS viermal gewaschen und danach mittels Photoimager bzw. Laserscanner ausgewertet. Alle 
Inkubations- und Waschschritte erfolgen unter leichter Bewegung der Streifen in einer Inkubationswanne auf einem Laborschüttler. Bei der Auswertung und Dokumentation der Nitrocellulosestreifen wurde zunächst eine Bestrahlung mittels visuellem Licht (vis.) und danach mittels UV-Licht (uv) zur Anregung der Fluoreszenz (FITC) vorgenommen. Mittels des (vis.)-Bildes ist eine Quantifizierung des Magnetits in den Präparationen möglich. Eine grüne Fluoreszenz der (uv)-Bilder zeigt die Bindung des Antikörpers (biologische Aktivität) quantitativ auswertbar an.

\subsection{Glutaraldehydaktivierung der Magnetit- HSA-Partikel}

Zur Kopplung weiterer Proteine an Magnetit-HSA-Partikel wurden mittels Oxalsäure aktivierte Magnetit-HSA-Partikel mit Glutaraldehyd aktiviert. Dazu wurden Aliquots der Partikel-Präparation mit $1 \mathrm{ml} \mathrm{2,5 \%} \mathrm{(w/v)} \mathrm{Glutaraldehyd}$ vermischt, 1 Stunde bei Raumtemperatur leicht geschüttelt und anschließend dreimal mit destilliertem Wasser gewaschen.

\subsection{Kopplung von Protein A und Aktivitätstest}

Die unter 2.4 aktivierten Magnetit-HSA-Partikel wurden in $200 \mu \mathrm{l}$ destilliertem Wasser resuspendiert und mit $100 \mu \mathrm{g}$ Protein A ( $25 \mu \mathrm{l})$ zunächst 2,5 Stunden bei Raumtemperatur und anschließend über Nacht bei $4^{\circ} \mathrm{C}$ leicht geschüttelt. Nach Waschung mit destilliertem Wasser kann der Aktivitätstest durchgeführt werden. Der Aktivitätstest erfolgt wie unter 2.3 beschrieben als Festphasenassay auf Nitrocellulosestreifen. Als Nachweissonde wird ein FITC-fluoreszenzmarkierter Antikörper (Kaninchen) verwendet.

\subsection{Materialien}

Es wurden Chemikalien und Biochemikalien folgender Firmen verwendet:

$\mathrm{FeCl}_{2} \times 4 \mathrm{H}_{2} \mathrm{O}$ (Fluka), $\mathrm{FeCl}_{3} \times 6 \mathrm{H}_{2} \mathrm{O}$ (Riedel-deHaen), Ammoniak (Riedel-deHaen), Oxalsäure, Weinsäure und Citronensäure (Fluka), PBS (phosphate buffered saline) (Sigma), Glutaraldehyd (Sigma), HSA (Sigma), Protein A (ICN), Anti-HSA FITC-markiert (ICN), IgG-FITC-markiert (anti-Huhn, Kaninchen) (Sigma), Nitrocellulosemembranen 0,45 $\mu \mathrm{m}$ (Bio-Rad), Gelantine (Bio-Rad)

\section{Ergebnisse und Diskussion}

\subsection{Herstellung von Magnetit-Protein-Partikeln und chemische Aktivierung}

Magnetit-Protein-Partikel können durch direkte Kopräzipitation von Proteinen aus Eisensalzlösungen hergestellt werden. Als Modellprotein wurde HSA verwendet. Die primären Fällungsprodukte (HSA/M) weisen zwar einen hohen Magnetitgehalt (vgl. Abbildung 1 (vis)) auf, sind aber wie Magnetit-Präparationen (M, Negativkontrolle) biologisch inaktiv, da keine fluoreszenzmarkierten Antikörper gebunden werden können (vgl. Abbildung 1 (uv)). Die HSA-Verdünnungsreihe und der fluoreszenzmarkierte Antikörper (Positivkontrollen) liefern deutliche Fluoreszenzsignale. Erst nach Aktivierung mit organischen Säuren (vgl. z. B. Oxalsäure, Abbildung 1) gelingt der Nachweis von biologischer Aktivität (Bindung

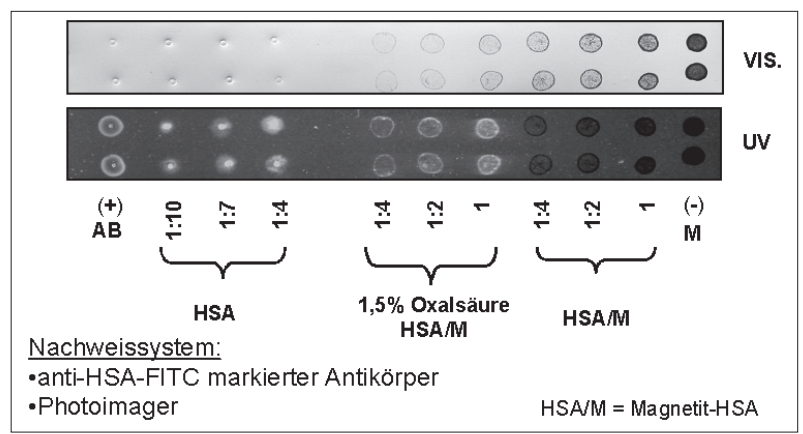

Abb. 1: Chemische Aktivierung von Magnetit-HSA-Partikeln

von anti-HSA Antikörpern). Obwohl die Magnetit-HSAPartikel deutlich weniger Magnetit (vgl. Abbildung 1 (vis.)) enthalten, sind diese sehr gut mittels Magnet aus Lösungen abscheidbar.

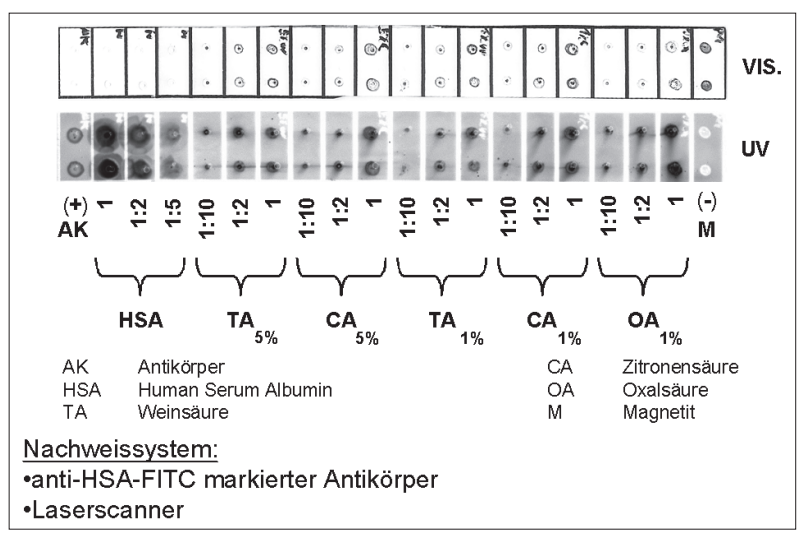

Abb. 2: Optimierung der chemischen Aktivierung für MagnetitHSA-Partikel

Die chemische Aktivierung kann mittels unterschiedlicher organischer Säuren (z. B. Zitronensäure, Weinsäure und Oxalsäure) erreicht werden (vgl. Abbildung 2). Die beschriebenen organischen Säuren wurden durch ein Screening unter Verwendung des von uns entwickelten Festphasenassays aus einer hohen Zahl von Kandidatenverbindungen selektiert. Optimale Ergebnisse erhält man mit Oxalsäure. Die optimale Oxalsäurekonzentration beträgt 1,5 \% (w/v). Es können Magnetit-HSA-Partikel mit hoher biologischer Aktivität und zur magnetischen Beweglichkeit ausreichend hohem Magnetitgehalt präpariert werden.

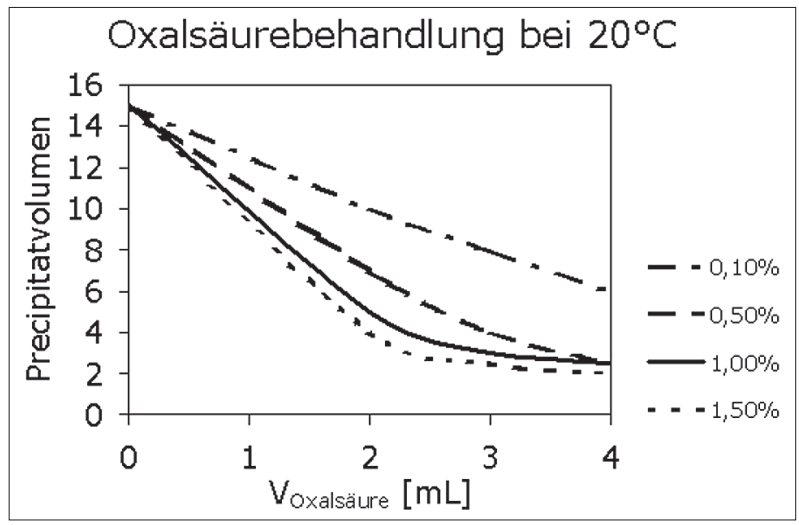

Abb. 3: Optimierung der Oxalsäurebehandlung

Die Konzentration und das zur Aktivierung verwendete Volumen der Oxalsäure, bezogen auf definierte Partikelzahlen, spielen bei der Aktivierung ebenfalls eine Rolle 
(vgl. Abbildung 3). Durch Verwendung unterschiedlicher Säurekonzentrationen (von 0,1-1,5\% (w/v)) kann man Magnetit-HSA-Partikel mit unterschiedlich hohem Magnetitgehalt erhalten. Bei dem Aktivierungsschritt sinkt das Volumen der Magnetit-HSA-Partikel durch Reduktion des Magnetitgehaltes beträchtlich. Reine Magnetit-Partikel werden unter diesen Reaktionsbedingungen vollständig aufgelöst.

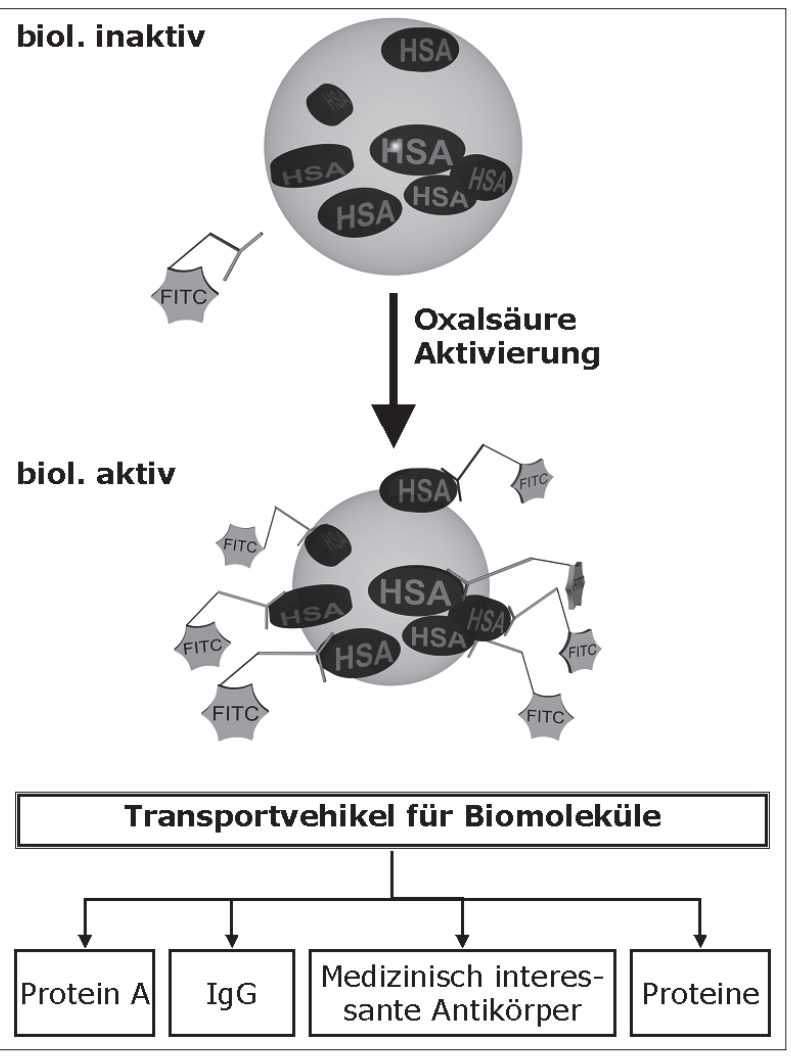

Abb. 4: Modell der chemischen Aktivierung

Die Ergebnisse der Experimente führen zu einer Modellvorstellung für die chemische Aktivierung (Abbildung 4). Während der chemischen Aktivierung werden zunächst biologisch inaktive Magnetit-HSA-Partikel, in denen die Erkennungsepitope für die anti-HSA-Antikörper (FITC-fluoreszenzmarkiert) durch Magnetit an der Partikeloberfläche verdeckt sind, in biologisch aktive Partikel überführt. Die organischen Säuren beseitigen Magnetit und legen Erkennungsepitope des HSA an der Oberfläche der Partikel frei. Dabei wird das Volumen der Partikel deutlich reduziert.

\subsection{Glutaraldehydaktivierung von Magnetit-HSA- Partikeln und Kopplung von Proteinen}

Magnetit-HSA-Partikel sind durch die an der Oberfläche der Partikel liegenden Proteinregionen sehr gut geeignet für Strategien zur kovalenten Kopplung von Biomolekülen mittel unterschiedlicher Kopplungsreagenzien. In Abbildung 5 sind die Optimierungsergebnisse einer zweistufigen Aktivierungsmethode von Magnetit-HSA-Partikeln mittels Glutaraldehyd schematisch zusammengefasst. Das Verfahren ermöglicht die kovalente Kopplung von Immunglobulinen (IgG) und Protein A. Für die präparierten Magnetit-HSA-Protein A-Partikel ist der Aktivitätstest unter Verwendung des Festphasenassays in Abbildung 6 dargestellt. Zur Kopplung werden relativ hohe Protein-

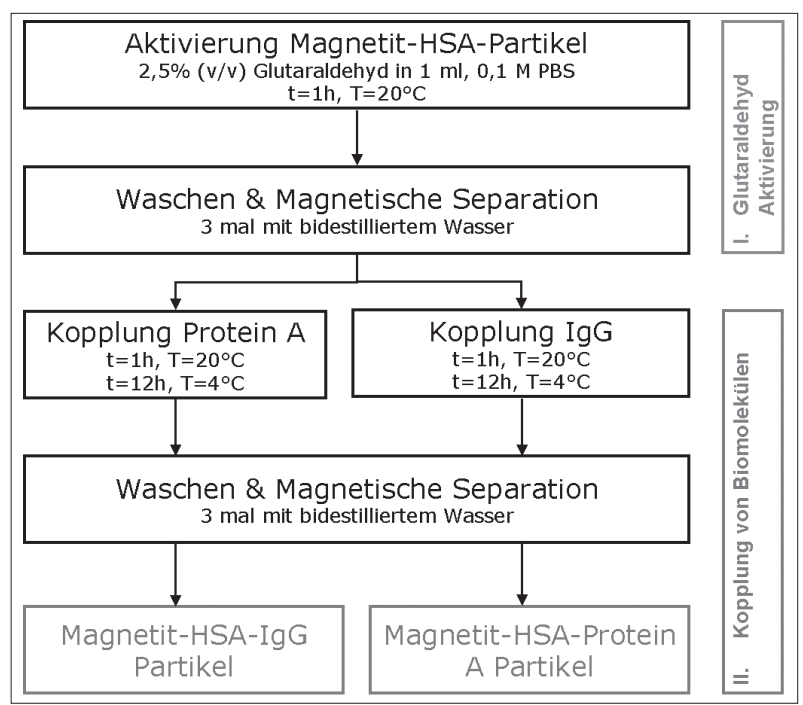

Abb. 5: Schema der Zweistufen-Glutaraldehyd-Aktivierung

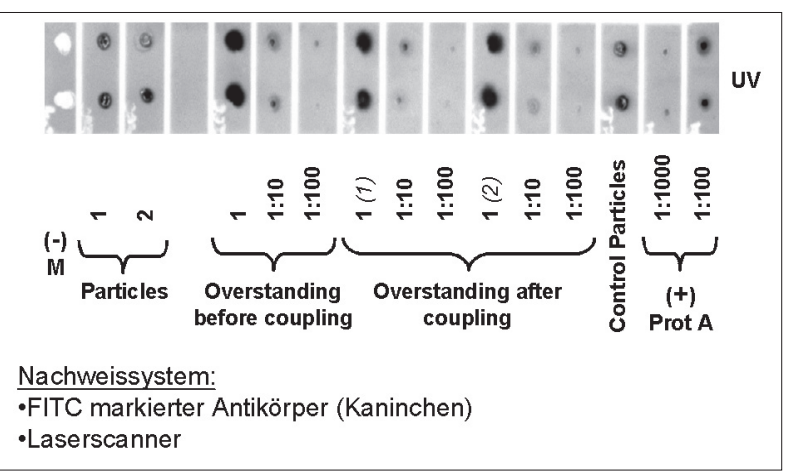

Abb. 6: Präparation und biologische Aktivität von Magnetit-HSAProtein A-Partikeln

konzentrationen (100 $\mu$ g pro $225 \mu \mathrm{l}$ Ansatz) benötigt, die nicht vollständig gekoppelt werden. Die Abbildung 6 zeigt die Überstandsanalyse von 2 Präparationen. Die Partikelpräparationen zeigen hohe biologische Aktivität des über Glutaraldehyd gekoppelten Protein A (Bindung von FITC-markierten Antikörpern über den Fc-Teil an Protein A). Als Negativkontrolle werden Magnetitpartikel, als Positivkontrollen eine Protein A Verdünnung und MagnetitHSA-Protein A-Kontrollpartikel verwendet. Magnetit-HSAProtein A-Partikel wurden zur Größenbestimmung mittels Atomic Force Mikroskopie untersucht (vgl. Abbildung 7). Es konnte eine Größe von ca. $93 \mathrm{~nm}$ bestimmt werden. Die Abbildung zeigt ein 3D-Bild eines Magnetit-HSA-Protein A-Nanopartikels auf einer Siliciumoberfläche.

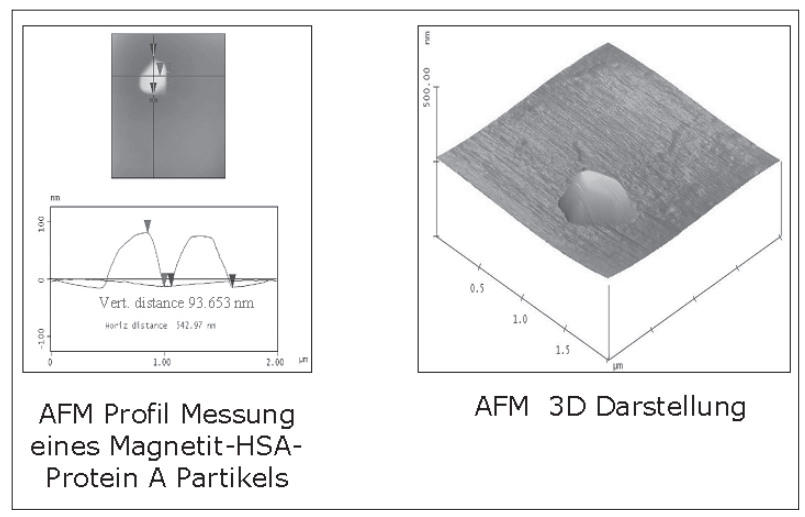

Abb. 7: Atomic Force Microscopy (AFM) 


\section{Zusammenfassung}

Das neue Präparationsverfahren ermöglicht die Präparation von biologisch aktiven Magnetit-Protein-Partikeln. Dazu werden die Proteine direkt aus einem Gemisch von Fe(II)- und Fe(III)-Salzen unter Verwendung von Ammoniak als Fällungsmittel kopräzipitiert. Die entstehenden Präzipitate sind zunächst biologisch inaktiv und müssen mittels eines zusätzlichen chemischen Reaktionsschrittes aktiviert werden. Unter den unterschiedlichsten, mittels Festphasenassay geprüften, Aktivierungsstrategien zeigten sich organische Säuren (z. B. Zitronensäure, Weinsäure und Oxalsäure) als effektivste Aktivierungmittel. Die chemische Aktivierung hängt von den Aktivierungsbedingungen (z. B. Temperatur und Konzentration der organischen Säuren) unter denen die primären Präzipitationsprodukte behandelt werden ab. Zur Präparation von biologisch hochaktiven Magnetit-HSA-Partikeln wurde die Einwirkung von 1,5\%-iger (w/v) Oxalsäure bei Zimmertemperatur als optimaler Aktivierungsschritt bestimmt.

Während der Säurebehandlung vergrößert sich die Protein/Magnetit-Rate sehr stark. Wahrscheinlich wird dabei oberflächlich auf dem Magnetit-Protein-Komplex abgelagertes Magnetit durch die organischen Säuren gelöst und Proteinstrukturen freigesetzt (Nachweis von Antikörper-Erkennungsepitopen mittels Festphasenassay). Die Untersuchungen lassen ein Modell der chemischen Aktivierung (vgl. Abbildung 4) postulieren, dass eine beachtliche Volumenreduktion der primären Präzipitationsprodukte während des chemischen Aktivierungsschrittes zeigt. Nach diesem Schritt sind Proteinregionen an der Oberfläche der Partikel frei zugängig und werden dadurch nachweisbar (z. B. durch fluoreszenzmarkierte (FITC) Antikörper).

Die biologische Aktivität der Magnetit-Protein-Komplexe wurde mittels eines neu entwickelten Festphasenassays unter Verwendung von Nitrocellulosemembranen als Trägermaterial und fluoreszenzmarkierten Antikörpern als Nachweisreagens bestimmt. Der Test ermöglicht die parallele Testung einer Vielzahl unterschiedlicher Proben, einschließlich Positiv- und Negativkontrollen, im Screeningverfahren. Der Test ist automatisierbar.

Chemisch aktivierte Magnetit-HSA-Partikel sind favorisierte magnetische Partikel für die chemische Kopplung von unterschiedlichen Biomolekülen. Sie könnten als generell einsetzbare Vehikel zur Bindung und zum Transport von biologisch oder medizinisch interessanten Molekülen wie Protein A, Immunglobulinen (IgG), medizinisch interessanten Antikörpern und anderen Proteinen verwendet werden (vgl. Abbildung 4). Die chemische Kopplung von Biomolekülen an die präparierten Magnetit-HSA-Partikel gelingt z. B. mittels Glutaraldehyd. Magnetit-HSA-Protein A-Partikel, die durch direkte Präzipitation von Magnetit-HSA-Partikeln, chemische Aktivierung und ein zweistufiges Glutaraldehydverfahren zur kovalenten Kopplung von Protein A präpariert wurden, könnten als generell verwendbares Transportvehikel zum Transport von Protein A-bindungsfähigen Antikörpern in biologischen Systemen verwendet werden.

Magnetit-HSA-Protein A-Nanopartikel mit hohen IgG-Bindungskapazitäten wurden präpariert und mittels Atomic Force Mikroskopie (AFM) charakterisiert. Sie besitzen eine Größe von ca. 93 nm.

\section{Literatur}

[1] Y.J. Wang, S.M. Hussein, G.P. Krestin, Eur. Radiol. 11 (2001) 2319.

[2] C. Alexiou, W. Arnold, R.J. Klein, et al., Cancer Res. 60 (2000) 6641.

[3] A. Ito, K. Tanaka, T. Kobayashi, et al.,Cancer Sci. 94 (2003) 308.

[4] M. Levin, N. Carlesso, R. Weissleder, et al., Nature Biotech. 18 (2000) 410.

[5] A.E. Merbach, E. Toth (Eds.), The Chemistry of Contrast Agents in Medicinical Magnetic Resonance Imaging, Wiley Chichester, 2001.

[6] R. Weissleder, D.D. Stark, B.L. Engelstad, et al.,Am. J. Roentgenol. 152 (1989) 167.

[7] Q.A. Pankhurst, J. Conolly, et al., J. Phys. D 36 (2003) R167.

[8] P. Tartaj, M.P. Morales, et al., J. Phys. D 36 (2003) R182.

[9] C.C. Berry, A.S.G. Curtis, J. Phys. D 36 (2003) R198.

[10] U. Häfeli, W. Schütt, J. Teller, M. Zborowski, Scientific and Clinical Applications of Magnetic Carriers, Plenum Press, Nerw York, 1997.

[11] L. Babes, B. Denizot, G. Tanguy, et al., J. Colloid Interface Sci. 212 (1999) 474.

[12] R. Weissleder, A. Bogdanov, et al., Adv. Drug Delivery Rev. 16 (1995) 321.

[13] R. S. Molday, D. Mackenzie, J. Immunol. Methods 52 (1982) 353.

[14] Y. Zhang, N. Kohler, M. Zhang, Biomaterials 23 (2002) 1553.

[15] C. W. Jung, Magn. Reson. Imaging 12 (1995) 675.

\section{Danksagung}

Die Verfasser danken Herrn R. Ries (TFH Wildau, Physikalische Technik) für die Größenbestimmung der Partikel mittels AFM und Herrn M. Springer (TFH Wildau, Ingenieurwesen/Wirtschaftsingenieurwesen) für die graphische Gestaltung der Abbildungen.

\section{Autoren}

Dr. sc. nat. Hans-Dieter Hunger

Technische Fachhochschule Wildau

Fachbereich Ingenieurwesen/Wirtschaftsingenieurwesen Tel. +49 3375 508-459

hdhunger@igw.tfh-wildau.de

Dipl. Biochem. Katerina Vaskova

University Ss. "Cyril and Methodius", 1000 Skopje, Republic of Macedonia 